\title{
Assessment of salivary and serum antioxidant status in patients with recurrent aphthous stomatitis
}

\author{
Jalil Momen-Beitollahi ${ }^{1}$, Arash Mansourian ${ }^{1}$, Fatemeh Momen-Heravi ${ }^{2}$, Masoud Amanlou ${ }^{3}$, Stoyana Obra- \\ dov $^{2}$, Mahnaz Sahebjamee ${ }^{4}$
}

\footnotetext{
${ }^{1}$ Assistant Professor, Department of Oral Medicine and Dental Research Center, Dental School. Tehran university of Medical Sciences, Tehran, Iran

${ }^{2}$ Dentist, Dental School, Tehran University of Medical Sciences, Tehran, Iran

${ }^{3}$ Professor,Department of Medicinal Chemistry, Faculty of Pharmacy and Pharmaceutical Sciences Research Center, Tehran University of Medical Sciences, Tehran, Iran

${ }^{4}$ Professor, Department of Oral Medicine and Dental Research Center, Dental School. Tehran university of Medical Sciences, Tehran, Iran
}

\author{
Correspondence: \\ Department of Oral Medicine, Dental School \\ Tehran University of Medical Sciences \\ Tehran, Iran \\ amansourian@sina.tums.ac.ir
}

Received: 03/06/2009

Accepted: $23 / 12 / 200$

\begin{abstract}
Momen-Beitollahi J, Mansourian A, Momen-Heravi F, Amanlou M, Obradov S, Sahebjamee M. Assessment of salivary and serum antioxidant status in patients with recurrent aphthous stomatitis. Med Oral Patol Oral Cir Bucal. 2010 Jul 1;15 (4):e557-61.

http://www.medicinaoral.com/medoralfree $01 / \mathrm{v} 15 \mathrm{i} 4 /$ medoralv15i4p557.pdf
\end{abstract}

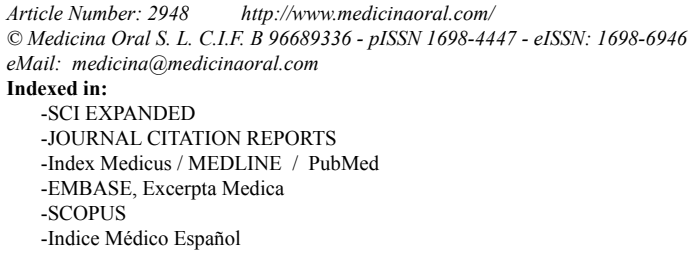

-Indice Médico Español

\begin{abstract}
Objective: The aim of this study was to determine the possible association of oxidant/antioxidant status and recurrent aphthous stomatitis (RAS).

Study design: The study consis ted of thirty-one patients with RAS and thirty-two healthy controls from whom saliva and blood samples were collected. Superoxide dismutase (SOD), glutathione peroxidase (GSHPx) and catalase (CAT) were measured in erythrocytes and total antioxidant status (TAS) was measured in plasma and saliva.

Results: Erythrocyte SOD activity was significantly lower in RAS patients in comparison to healthy controls $(\mathrm{P}=0.012)$. No significant differences were found in erythrocyte GSHPx, CAT activities, and salivary and plasma TAS between RAS patients and control subjects $(\mathrm{p}>0.1)$.

Conclusion: Changes in SOD activity may be important in the inflammatory reactions observed in RAS, but other tested defense systems such as CAT and GSHPx do not seem to play a primary role in the aetiopathogenesis of RAS. Moreover, the antioxidant system in saliva and plasma is not as affected as in erythrocytes in RAS patients, and therefore it may not be considered an appropriate indicator of the body's total antioxidant status.
\end{abstract}

Key words: Antioxidants, recurrent aphthous stomatitis, oxidative stress, saliva, superoxide dismutase. 


\section{Introduction}

Recurrent aphthous stomatitis (RAS) is a common but poorly understood oral mucosal disorder that affects almost $20 \%$ of population worldwide $(1,2)$. Clinical picture of RAS is defined by small, shallow, round or ovoid ulcerations, with well defined and circumscribed margins and with an erythematous halo around it. Ulcerations may be single or multiple and there have been described three types of aphthous ulcerations: minor, major and herpetiform. RAS occurs at intervals of few months to few days and heal spontaneously. It appears that it has some female predominance, white people are more affected than black people and children of higher socioeconomic status are more commonly affected than those of lower socioeconomic groups (2-4).The exact cause of RAS has not been disclosed clearly. However, some etiologic and predisposing factors such as trauma, stress, genetic, hypersensitivity, nutrition, immune disturbance, and hormonal imbalance are suggested (1-4). All of the above mentioned conditions can disturb the oxidant/ antioxidant balance of organism and can accelerate the formation of free radicals. Oxidative stress occurs when the intracellular concentrations of reactive oxygen radicals $\left(\mathrm{O}_{2} \mathrm{H}_{2} \mathrm{O}_{2}\right.$ and $\left.\mathrm{H}_{2} \mathrm{O}_{2}\right)$ increase over the physiologic values. The cytotoxic effects of free radicals are detrimental for mammalian cells, and lead to cell damage through its damaging effects on peroxoidation of double-chain fatty acids, protein and DNA, as well as, increase oxidative stress $(3,4)$.

Accordingly, mammalian cells have developed complicated antioxidant defense systems to prevent oxidative damage and to allow survival in an aerobic environment. This system includes enzymatic activities such as erythrocyte superoxide dismutase (SOD), catalase (CAT), glutathione peroxidase (GSHPx), blood antioxidant molecules as well as non-enzymatic antioxidants including vitamins A,C, E melatonin, and uric acid (UA) (5-8).

It has been reported in few studies that impaired oxidant/ antioxidant balance is responsible for the tissue damage in RAS patients (7-9). Regarding the insufficient studies pertinent to this issue, the aim of the present study was to measure some parameters of oxidative stress including erythrocyte superoxide dismutase, catalase, glutathione peroxidase, and the level of salivary and blood oxidant/antioxidant status of RAS patients and compare it with healthy subjects to find new information about RAS aetiopathology.

\section{Materials and Methods \\ Patients}

The institutional review board and Ethics Committee of Tehran University of Medical Sciences approved the study protocol and each subject signed a detailed informed consent form. The study included 32 RAS cases who applied to outpatient clinic of oral medicine department of Tehran University of Medical Sciences and 32 healthy individuals who age and sex were matched. The RAS patients had oral ulcer attack recurring at least three times a year and had active lesions during the study $(1,10)$. RAS was diagnosed clinically by an expert in oral medicine. Patients were otherwise healthy and had active aphthous lesions during the study. They were not under a therapeutic regimen for the past 3 months. The following laboratory tests were performed; erythrocyte sedimentation rate, blood cell counts and indexes; electrolytes, glucose, liver and kidney function tests, UA, calcium, phosphorus, globulin, albumin, iron in blood, vitamin B12 and folic acid. The patients with normal biochemical analysis mentioned above were participated into the study. Patients with Behçet's disease, chronic diarrhea, trauma history, any systemic disease, smoking history, alcohol drinking, or addiction were not included in the study.The control group included 32 self-admitted healthy individuals that were either medical students or clinic staffs, who denied having RAS or using medications or vitamins.

\section{Measurements}

Analyses were carried out in saliva, plasma and erythrocytes. Superoxide dismutase (SOD), catalase (CAT) and glutathione peroxidase (GSHPx) of erythrocyte, and total antioxidant status (TAS) of saliva and plasma, were measured. Activities of SOD, CAT and GSHPx in erythrocytes are expressed in international units, and TAS in saliva and plasma is expressed as $\mathrm{mmol} / \mathrm{L}$ of plasma and saliva respectively.

\section{Plasma preparation}

Fasting venous blood samples $(5 \mathrm{ml})$ from 31 patients and 32 healthy control individuals were drawn into vacutainers containing heparin as anticoagulant. Samples were centrifuged at $3000 \mathrm{~g}$ for $10 \mathrm{~min}$ at $4{ }^{\circ} \mathrm{C}$. Plasma was separated and buffy coat was discarded by aspiration. Erythrocytes were washed four times with cold physiological saline and stored at $-40{ }^{\circ} \mathrm{C}$ until analysis.

\section{Saliva preparation}

Samples were gained in the morning following an overnight fast. The patients were first asked to rinse their mouth using distilled water. After $5 \mathrm{~min}$, we started to gain unstimulated saliva samples. Then the patients were told to sit comfortably and to spit into the plastic tubes five times per min for $5 \mathrm{~min}$. Samples were centrifuged $4000 \mathrm{~g}$ for $10 \mathrm{~min}$ at $4{ }^{\circ} \mathrm{C}$, the upper parts were drawn and stored in small aliquots at $-40{ }^{\circ} \mathrm{C}$.

Superoxide dismutase (SOD) and glutathione peroxidase (GSHPx) activity

The SOD and GSHPx activities of erythrocytes were estimated for the hemolyzsates by the use of commercially available kits RADSOD and RANSEL (Randox Laboratory Ltd., Ardmore, UK). The erythrocytes were hemo- 
lysed by the addition of ice-cold deionized water and vigorously vortexed. SOD estimation was based on the generation of superoxide radicals produced by xanthine and xanthine oxidase, which reacts with 2-(4-iodophenyl)-3 -(4-nitrophenol)-5-phenyl tetrazoliu-chloride (INT) to form a red formazan dye. The SOD activity was measured by the degree of inhibition of this reaction.

Erythrocyte GSHPx determination was based on the following principle: GSHPx catalyzes the oxidation of glutathione by cumene hydroperoxide. In the presence of glutathione reductase and reduced nicotinamide adenine dinucleotide, phosphate (NADPH) the oxidized glutathione (GSSG) is immediately converted to the reduced form with a contaminant oxidation of NADPH to NADP+. The decrease in absorbance at $340 \mathrm{~nm}$ was measured by a spectrophotometer $(11,12)$.

Erythrocyte catalase activity (CAT)

The CAT activity was measured in samples by method applied by Cimen et al. (8). The decomposition of the substrate $\mathrm{H}_{2} \mathrm{O}_{2}$ was monitored spectrophotometrically at $240 \mathrm{~nm}$. Specific activity was determined as micromole substrate decomposed per minute per milligram of protein (i.e. U/mg protein).

Plasma and salivary total antioxidant status (TAS)

The total antioxidant status of the plasma and saliva were measured using a recent automated colorimetric measurement method for the TAS developed by Erel (13).

In this method the hydroxyl radical, the most potent biological radical, is created by the Fenton reaction, and reacts with the colourless basis odianisidine to produce the dianisyl radical, which is bright yellowish-brown in colour. Upon the addition of a plasma and saliva samples, the oxidative reactions initiated by the hydroxyl radicals present in the reaction mix are suppressed by the antioxidant components of the plasma and saliva, hampering the colour change and thereby providing an effective measure of the total antioxidant capacity of the plasma and saliva. The analysis results are expressed as micromolar Trolox equivalent per liter (mmol Trolox eq./L), and the precision of this assay is excellent, being lower than 3\% 9 (14).

\section{Statistical analysis}

All values were expressed as mean \pm SD. Statistical analysis was performed by using chi-square and leven's test for equality of variances. For statistical analysis independent sample t-test and one-sample Kolmogrov Smirnov test were also used. Statistical significance was set at $\mathrm{P}<0.05$.

\section{Results}

The patient group comprised 20 females and 11 males, whose mean age was $28.13 \pm 8.05$ years, while the control group consisted of 21 females and 11males, whose mean age was $27.09 \pm 7.54$ years. The SOD, GSHPx, CAT in erythrocytes as well as plasma and salivary TAS values of RAS patients and controls are presented in table 1.

As seen in table 1, the comparison between the patient group and the control group showed that erythrocyte SOD activity was significantly lower in the former ( $\mathrm{P}=0.012)$, but there was not a statistically significant difference between two groups in erythrocyte CAT ( $\mathrm{P}=0.769)$, erythrocyte GSHPx $(\mathrm{P}=0.721)$, salivary TAS $(\mathrm{P}=0.971)$ and plasma TAS $(\mathrm{P}=0.311)$. The comparison between the TAS level in plasma and saliva in RAS group and the control group revealed that the level of TAS in plasma was significantly higher than saliva $(\mathrm{P}<0.01)$.

Table 1. Erythrocyte superoxide dismutase (SOD), catalase (CAT), glutathione peroxidase (GSHPx) activities and total antioxidant status (TAS) levels in plasma and saliva of RAS patients and control subjects.

\begin{tabular}{|l|c|c|c|c|c|}
\hline & $\begin{array}{c}\text { SOD } \\
\text { (U/mg } \\
\text { protein) }\end{array}$ & $\begin{array}{c}\text { CAT } \\
\text { (U/mg } \\
\text { protein) }\end{array}$ & $\begin{array}{c}\text { GSHPx } \\
\text { (U/mg protein) }\end{array}$ & $\begin{array}{c}\text { TAS } \\
\text { (mmol/L } \\
\text { plasma) }\end{array}$ & $\begin{array}{c}\text { TAS } \\
\text { (mmol/L } \\
\text { Saliva) }\end{array}$ \\
\hline RAS & $1131.03 \pm$ & $26.96 \pm$ & $9515.09 \pm$ & $1.31 \pm$ & $1.08 \pm$ \\
$(\mathrm{n}=31)$ & 341.25 & 6.98 & 3778.85 & 0.17 & 0.26 \\
\hline Control & $1417.68 \pm$ & $27.56 \pm$ & $9154.06 \pm$ & $1.36 \pm$ & $1.08 \pm$ \\
$(\mathrm{n}=32)$ & 515.91 & 8.94 & 4199.70 & 0.20 & 0.34 \\
\hline P-value & $0.012^{*}$ & 0.769 & 0.721 & 0.311 & 0.971 \\
\hline
\end{tabular}

SOD, superoxide dismutase; CAT, catalase; GSHPx, glutathione peroxidase; TAS, total antioxidant status. Values are expressed as mean \pm SD. * statistical significance 


\section{Discussion}

Considerable activity of reactive oxygen radicals may lead to destroyed normal cell functions and integrity of cell structures. Oxidative stress in biological systems can be induced by the consumption of antioxidants and/ or by an overload of oxidant species, so that antioxidant levels become deficient. It appears that imbalance between free radicals and antioxidants causes many inflammatory oral soft tissue diseases varying from infections and immunologic diseases to lethal cancers $(4,6,8)$. In spite of a great deal of effort spent on this subject, few studies were reported in the literature investigating the possible relation between RAS and oxidant/ antioxidant pathway (7-9).

The organism has enzymatic (e.g. superoxide dismutase, catalase, glutathione peroxidase) and non-enzymatic (e.g. vitamin C, vitamin E) antioxidant defense system that work as scavenger for this harmful reactive oxygen radicals. Radical scavenging antioxidants are utilized by the increased free-radical activity associated with several conditions, and the amount of each antioxidant response has been used to indirectly evaluate free-radical activity (15-17).

In the present study, we investigated whether there is any alteration in SOD, GSHPx and CAT activity in erythrocytes and TAS activity in plasma and saliva in RAS cases when compared with those of the control group. We found a slight decrease in CAT, a modest increase in GSHPx activity and unchanged activity of TAS. Only SOD activity altered significantly $(\mathrm{P}=0.012)$.

SOD is an enzyme that catalyzes the dismutation of two superoxide anions $\left(\mathrm{O}_{2}-\right)$ into hydrogen peroxide and molecular oxygen. Superoxide dismutase defends the tissue to a certain degree from the harmful effects of superoxide radicals. There are two forms of SOD, Cu-Zn-SOD and Mn-SOD. A decreased level of SOD activity may be due to high consumption, and lead to overproduction of $\mathrm{H}_{2} \mathrm{O}_{2}$ that is a product of dismutation reaction. CAT hydrolyzes $\mathrm{H}_{2} \mathrm{O}_{2}$ into $\mathrm{H}_{2} \mathrm{O}$ and $1 / 2 \mathrm{O}_{2}$ and recognized to be secondary antioxidant enzyme in peroxidative defense. In view of the fact that GSHPx is the dominant antioxidant protective element participating in getting rid of $\mathrm{H}_{2} \mathrm{O}_{2}$ in the site, the increased amount of it may be due to genetic control mechanisms and feedback effects of $\mathrm{H}_{2} \mathrm{O}_{2}$ on mRNA expression $(18,19)$.

Moreover, we found slightly decreased blood TAS in RAS patients but we could not find any significant difference between salivary TAS of RAS patients and controls. Although it was shown that only SOD is significantly different in two groups, other values may show such difference with a larger sample size in further studies. So, it can be concluded that all of these changes and differences somehow the result of oxidant nature of the disease and again emphasizes such pathogenesis in patients with RAS.

Cimen et al. (8) measured SOD, GSHPx, and CAT activity in plasma and erythrocytes of patients with RAS and compared it with control group. They observed a relative reduction in CAT and GSHPx activity and AOP in erythrocytes of RAS patients and finally concluded that enzymatic and non enzymatic antioxidant defenses in RAS patients are defective. Karincaoglu et al. (7) evaluated plasma and saliva antioxidant activity in patients with RAS and reported that SOD activity in plasma of RAS patients was lower than of control group $(\mathrm{P}<0.001)$. But Gunduz et al. (20) obtained exactly opposite results. These relative controversies among studies may be associated with several factors including: sample size variations, application of various methods and genetic divergence of each population. Furthermore, the composition and antioxidant capacity may vary by dietary and nutrient deficiency.

In recent years, there are increasing reports on literature regarding application of natural anti oxidant products on management of RAS. These herbal preparations including extracts and/or essential oils of medicinal plants exhibits promising effects on shortening healing time and severity of pain in RAS patients (1). On the other hand, food, dietary and nutrient deficiency has important role in human health and as an initiating factor in RAS (1-3). Among these material antioxidant vitamins and micro-elements such as zinc and selenium considered more attention because they are involved in antioxidant defense system (8).

It can be concluded that antioxidant system in saliva and plasma is not as affected as in erythrocytes in RAS patients and therefore it may not be considered an appropriate indicator of total antioxidant status of the body. Nevertheless, neither present study nor previous ones could categorically conclude, either is it that the antioxidant system impairment in RAS patients causing aphthae, or vice versa, the full antioxidant impairment in aphthous patient is the consequence of aphthous ulcers. We propose that future studies should be controlled, multicenter prospective studies, which shall include large case series and this issue should be conducted. The results may be very useful in founding new treatments using antioxidant supplementation or nutritional prescriptions that might elevate antioxidant system level in RAS patient.

\section{References}

1. Babaee N, Mansourian A, Momen-Heravi F, Moghadamnia A, Momen-Beitollahi J. The efficacy of a paste containing Myrtus communis (Myrtle) in the management of recurrent aphthous stomatitis: a randomized controlled trial. Clin Oral Investig. 2010;14:65-70.

2. Ship JA. Recurrent aphthous stomatitis. An update. Oral Surg Oral Med Oral Pathol Oral Radiol Endod. 1996;81:141-7.

3. Porter SR, Scully C, Pedersen A. Recurrent aphthous stomatitis. Crit Rev Oral Biol Med. 1998;9:306-21.

4. Scully C, Gorsky M, Lozada-Nur F. The diagnosis and manage- 
ment of recurrent aphthous stomatitis: a consensus approach. J Am

Dent Assoc. 2003;134:200-7.

5. Chambers JC, Haskard DO, Kooner JS. Vascular endothelial function and oxidative stress mechanisms in patients with Behçet's syndrome. J Am Coll Cardiol. 2001;37:517-20.

6. Beevi SS, Rasheed AM, Geetha A. Evaluation of oxidative stress and nitric oxide levels in patients with oral cavity cancer. Jpn J Clin Oncol. 2004;34:379-85.

7. Karincaoglu Y, Batcioglu K, Erdem T, Esrefoglu M, Genc M. The levels of plasma and salivary antioxidants in the patient with recurrent aphthous stomatitis. J Oral Pathol Med. 2005;34:7-12.

8. Cimen MY, Kaya TI, Eskandari G, Tursen U, Ikizoglu G, Atik U. Oxidant/antioxidant status in patients with recurrent aphthous stomatitis. Clin Exp Dermatol. 2003;28:647-50.

9. Cağlayan F, Miloglu O, Altun O, Erel O, Yilmaz AB. Oxidative stress and myeloperoxidase levels in saliva of patients with recurrent aphthous stomatitis. Oral Dis. 2008;14:700-4.

10. Albanidou-Farmaki E, Deligiannidis A, Markopoulos AK, Katsares V, Farmakis K, Parapanissiou E. HLA haplotypes in recurrent aphthous stomatitis: a mode of inheritance? Int J Immunogenet. 2008;35:427-32.

11. Battin EE, Brumaghim JL. Antioxidant activity of sulfur and selenium: a review of reactive oxygen species scavenging, glutathione peroxidase, and metal-binding antioxidant mechanisms. Cell Biochem Biophys. 2009;55:1-23.

12. Durmaz A, Dikmen N. Homocysteine effects on cellular glutathione peroxidase (GPx-1) activity under in vitro conditions. J Enzyme Inhib Med Chem. 2007;22:733-8.

13. Erel O. A new automated colorimetric method for measuring total oxidant status. Clin Biochem. 2005;38:1103-11.

14. Cao G, Prior RL. Comparison of different analytical methods for assessing total antioxidant capacity of human serum. Clin Chem. 1998:44:1309-15.

15. Lih-Brody L, Powell SR, Collier KP, Reddy GM, Cerchia R, Kahn E, et al. Increased oxidative stress and decreased antioxidant defenses in mucosa of inflammatory bowel disease. Dig Dis Sci. 1996;41:2078-86.

16. Sezer E, Ozugurlu F, Ozyurt H, Sahin S, Etikan I. Lipid peroxidation and antioxidant status in lichen planus. Clin Exp Dermatol. 2007;32:430-4.

17. Hoelzl C, Bichler J, Ferk F, Simic T, Nersesyan A, Elbling L, et al. Methods for the detection of antioxidants which prevent age related diseases: a critical review with particular emphasis on human intervention studies. J Physiol Pharmacol. 2005;56 Suppl 2:49-64.

18. Miao L, St Clair DK. Regulation of superoxide dismutase genes: implications in disease. Free Radic Biol Med. 2009;47:344-56.

19. Dalton TP, Shertzer HG, Puga A. Regulation of gene expression by reactive oxygen. Annu Rev Pharmacol Toxicol. 1999;39:67-101.

20. Gunduz K, Ozturk G, Sozmen EY. Erythrocyte superoxide dismutase, catalase activities and plasma nitrite and nitrate levels in patients with Behçet disease and recurrent aphthous stomatitis. Clin Exp Dermatol. 2004;29:176-9.

Acknowledgments

This work was supported by a grant from the Research Council of the Tehran University of Medical Sciences. 